\title{
Impact of endoscopic duodenal biopsy on the detection of small intestinal villous atrophy
}

\author{
S.H. Saverymuttu, J. Sabbat, Margaret Burke' and J.D. Maxwell
}

Departments of Medicine and 'Histopathology, St. George's Hospital Medical School, London SWI7 ORE, UK

\begin{abstract}
Summary: Coeliac disease is underdiagnosed partly because of insufficient recognition of more subtle presentations and partly because of the relative difficulty of jejunal biopsy. We have compared new case detection of small intestinal villous atrophy in the 5 years following changing to endoscopic multiple biopsy with our preceding 9 year experience utilizing jejunal capsule suction biopsy. The detection rate for small intestinal disease doubled while the number of patients investigated by small bowel biopsy increased threefold. We recommend that endoscopic duodenal biopsy replace jejunal biopsy for routine diagnostic purposes.
\end{abstract}

\section{Introduction}

Diagnosis of small intestinal mucosal disorders is often delayed, ${ }^{1}$ and it is likely that many patients with coeliac disease remain undetected. ${ }^{2}$ This may be both because of the changing presentation of the disease with insufficient recognition of the more subtle non-gastroenterological manifestations, and because of the relative difficulty of small intestinal intubation and biopsy with the jejunal biopsy capsule ${ }^{1-3}$ Following the validation of endoscopic distal duodenal biopsy in the diagnosis of small intestinal villous atrophy, ${ }^{3}$ we compared the efficacy of this technique in the detection of new cases of small intestinal villous atrophy, with our previous experience utilizing jejunal capsule suction biopsy.

\section{Methods and results}

We have reviewed all patients from a general medical/gastroenterology firm at St. George's Hospital who underwent small intestinal biopsy between 1975 and 1988 for the investigation of possible small intestinal mucosal disease. During this period the staffing, clinic arrangements and outpatient numbers remained stable. For the first 9 years jejunal capsule suction biopsy was the predominant mode of investigation, although in a few cases distal duodenal biopsies were obtained endoscopically. Following validation of multiple endoscopic duodenal biopsies in the diagnosis of villous atrophy, this technique has largely replaced jejunal biopsy, and for the past 5 years has been the

Correspondence: S.H. Saverymuttu, M.R.C.P.

Accepted: 24 July 1990 method of choice. A few cases were investigated by suction biopsy during the last 5 years, particularly when giardiasis was suspected, to allow jejunal juice aspiration. However, with more confidence in duodenal biopsies we have not performed suction biopsies over the last 3 years.

During the first 9 years the average number of jejunal biopsies performed annually was 11.6. (High numbers in 1979 and 1980 coincided with evaluation of an isotopic breath test for malabsorption ${ }^{4}$.) Endoscopic biopsies displaced jejunal biopsy in 1984, when 24 patients were investigated for possible small bowel disease and underwent multiple distal duodenal biopsies. The number of patients investigated in this way increased in each subsequent year to 78 in 1988 (Figure 1). The major part of this increase arose from a greater proportion of outpatients being investigated for relatively minor symptoms.

During the period when jejunal biopsy was the investigation of choice the annual new case detection rate for partial villous atrophy or subtotal villous atrophy varied between 0 and 4 per annum, with a mean of 1.9. The majority of these patients had 'classical' indications for biopsy (persistent diarrhoea, steatorrhoea, weight loss or macrocytic anaemia) but in $12.5 \%$ there was mild diarrhoea or just iron deficiency anaemia. Following the change to distal duodenal biopsy annual new case detection rate significantly increased to a mean of 4.6 $(P<0.05)$ with $37.5 \%$ having 'non-classical' indications for biopsy. This change was accompanied by a fall in positive biopsy rate from 1 in 7.5, to 1 in 10.3. For the 14-year period under review, there was a significant correlation between the total number of small bowel biopsies performed annually, and the number of new cases of small 


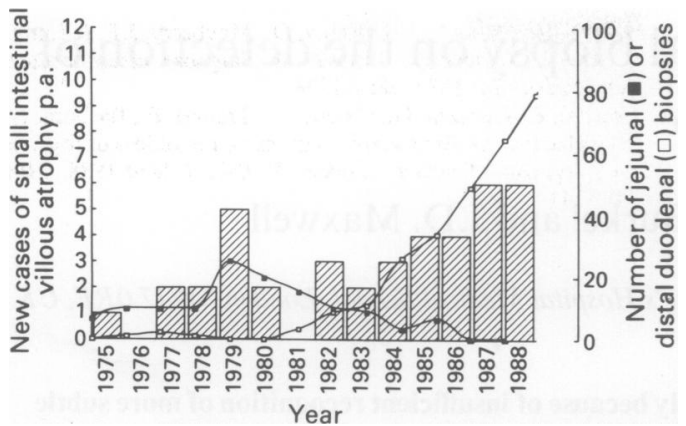

Figure 1 Number of cases of small intestinal villous atrophy diagnosed per year (hatched bars) versus number of jejunal ( $\square$ ) or distal ( $\square$ ) duodenal biopsies.

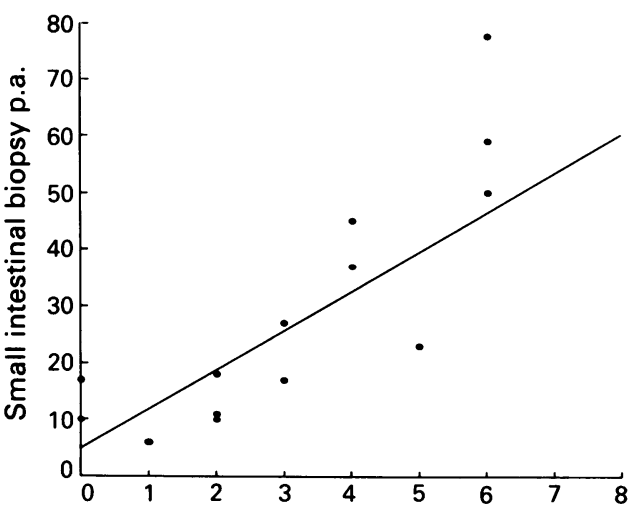

New cases of small intestinal villous atrophy p.a.

Figure 2 Significant correlation between total number of small bowel biopsies performed annually and new cases of small intestinal villous atrophy detected. $(r=0.79$, $P<0.05)$.

intestinal villous atrophy detected $(r=0.79$, $P<0.01$ ) (Figure 2). The majority of these cases fulfilled criteria for coeliac disease. Two patients had a final diagnosis of immunoproliferative small intestinal disease (IPSID), and one had infestation with strongyloides.

\section{Discussion}

The prevalence rate for coeliac disease in Northern Europe is reported to vary between 1:300 (west of
Ireland), 1:1000 (Sweden) and 1:1050 (United Kingdom). However, small intestinal mucosal lesions typical of coeliac disease have been reported in 1:266 apparently healthy Swedish blood donors, indicating that this disease is more common than previously suspected. ${ }^{5}$ Despite the growing awareness that the majority of patients with coeliac disease do not have severe symptoms associated with malabsorption, it has been estimated that diagnosis is often delayed by many years, and at least a quarter of patients remain undetected. ${ }^{1,2}$ Since these patients are denied treatment with a gluten-free diet they remain at higher risk for developing gut malignancy. ${ }^{6}$

Although various screening tests have been described (intestinal permeability; antigliadin antibodies), the diagnosis of coeliac disease and other mucosal disorders requires histological confirmation. Jejunal biopsy using suction biopsy capsule used to be the gold standard, but requires radiological screening, and can be time consuming and frustrating for patient and operator. ${ }^{3}$ Although various techniques have been developed to facilitate jejunal biopsy, endoscopy provides more ready access to the upper small bowel, and endoscopic distal duodenal biopsy has been shown to be very satisfactory for the routine diagnosis of small intestinal mucosal disorders, ${ }^{3}$ and can also be adequate for biochemical studies on tissue. ${ }^{7} \mathrm{~A}$ recent study has demonstrated that absent or markedly decreased duodenal folds at routine endoscopy were associated with a high incidence of subtotal villous atrophy. ${ }^{8}$ However, this approach cannot be recommended as an alternative to duodenal biopsy since a high proportion of patients with partial villous atrophy have normal endoscopic appearances of the duodenum.

Our experience confirms the practicality and ease of this technique which allows a more liberal approach to investigation of suspected malabsorption, and more than doubled the detection rate for new cases of small intestinal villous atrophy. Despite some increase in the workload of the histopathology department, we would recommend the wider application of endoscopic distal duodenal biopsy particularly in patients with iron deficiency anaemia where endoscopy has not revealed any bleeding lesion in the stomach, oesophagus or duodenal bulb. 
4. West, P.S., Levin, G.E., Griffin, G.E. \& Maxwell, J.D. Comparison of simple screening tests for fat malabsorption. $\mathrm{Br}$ Med J 1981, 282: 1501-1504.

5. Hed, J., Lieden, G., Ottoson, E., Strom, M., Walan, A. \& Grothy, O. IgA antigliadin antibodies and jejunal mucosal lesions in healthy blood donors. Lancet 1986, ii: 215.

6. Holmes, K.T., Prior, P., Lane, M.R., Pope, D. \& Allan, R.N. Malignancy in coeliac disease: effect of gluten free diet. Gut 1989, 30: 333-338.
7. Smith, J.A., Amoah, J., O'Reilly, D., Mayberry, J.F. \& Long, R. Use of endoscopic biopsies to diagnose disacchardase deficiencies. Gut 1987, 28: A1598.

8. Brocchi, E., Corazza, G., Caletti, G., Trggari, E., Barbara, L. \& Gasbarrini, G. Endoscopic demonstration of loss of folds in the diagnosis of coeliac disease. $N$ Engl $J$ Med 1988, 319: $741-744$ 